\title{
Paraovarian Hydatid Cyst Mimicking Acute Abdomen
}

\author{
Parvin Mostafa Gharabaghi ${ }^{1}$, Neda Shoari ${ }^{2}$, Azadeh Azadi ${ }^{2}$, Ali Dastran Tabrizi ${ }^{3}$, \\ Keivan Kashy Zonouzy ${ }^{4}$, Amir Vahedi ${ }^{5}$
}

Article History:
Received 9 October 2013
Accepted 7 January 2014
Revised 25 December 2013
Available online 7 January 2014
Keywords:
Cystadenoma
Echinococcosis
Paraovarian Cyst Hydatid
Corresponding Author:
Neda Shoari, Resident of Ob \& Gyn,
Tabriz University of Medical
Sciences, Tabriz, Iran.
Tel: +989143114622
Email: neda_4622@yahoo.com

\section{Article History:}

Revised 25 December 2013

Keywords:

Cystadenom

hinococcosis

Corresponding Author:

Neda Shoari, Resident of Ob \& Gyn

Tabriz University of Medical

\begin{abstract}
Echinococcosis is a parasitic disease that is produced by Echinococcus granulosus and still represents an important medical problem in many countries. Echinococcosis in extrahepatic sites is usually asymptomatic unless the cyst causes symptoms due to pressure, as in our case, or ruptures to the peritoneal cavity. Echinococcal cysts at unusual locations many times pose diagnostic dilemmas, and the diagnosis sometimes is made intraoperatively. Primary ovarian and paraovarian cyst hydatid disease is a very rare entity. In our case, a 25 -year-old female gravida 1 , para 1 , was admitted to the emergency department of the Alzahra Hospital of Tabriz due to diffuse abdominal pain, nausea, abdominal distension and urinary frequency of ten-day duration and exacerbation of pain from a few hours before admission. Clinical examination revealed tachycardia of 105 per minute, normal body temperature and normal blood pressure. Abdominopelvic sonography revealed a mass with regular thick contour located in the left ovary suggestive of huge cystadenoma or carcinoma of the ovary.

Emergent laparotomy was performed for suspected adnexal torsion. Intra operative findings were a large paraovarian mass with thick wall without any torsion. Mass was removed completely and sent for frozen section study. Pathological examination showed the diagnosis of hydatid cyst.

The postoperative period was uneventful and the patient was discharged 3 days later, with the advice to receive Albendazole. The patient referred to a general surgeon for management of the hepatic cysts.
\end{abstract}

1 - Professor of Ob \& Gyn, Women's Reproductive Health Research Center, Tabriz University of Medical Sciences, Tabriz, Iran.

2- Resident of Ob \& Gyn, Tabriz University of Medical Sciences, Tabriz, Iran.

3- Resident of General Surgery, Tabriz University of Medical Sciences, Tabriz, Iran.

4- Associate Professor of Pathology, Women's Reproductive Health Research Center, Tabriz University of Medical Sciences, Tabriz, Iran.

5- Associate Professor of Pathology, Department of Pathology, Tabriz University of Medical Sciences, Tabriz, Iran. 


\section{Introduction:}

Echinococcosis is a zoonosis that continues to be a major health problem in many countries. It is endemic in several countries in South America, the Middle and Far East, and around the Mediterranean Sea $(1,2)$. Hydatid disease is caused by the larval form of taenia echinococcus, which lives in the gut of dog, wild canines, and carnivorous animals that represent the definitive hosts (3). Humans become the accidentally intermediate hosts by ingesting taenia eggs. Then the slowly growing echinococcal cysts can achieve a volume of several liters and contain many thousands of protoscolices. With time daughter cysts can be formed. The liver is the organ most involved by echinococcosis (65\%-70\%) followed by the lungs (25\%). Less frequently hydatidosis involves the spleen, kidneys, peritoneum, brain, heart, and bones (4). Only few reports on abdominal hydatid disease simulating acute abdomen have been published to date. Primary ovarian and pararectal cyst hydatid are extremely rare in the literature a few cases have been reported.

Case Report :

A 25-year-old female gravida 1, para 1, was admitted to the emergency department of the Alzahra Hospital of Tabriz due to diffuse abdominal pain, nausea, abdominal distension and urinary frequency of ten-day duration and exacerbation of pain from a few hours before admission. Her medical history was free and no recent trauma was reported. Clinical examination revealed tachycardia of 105 per minute, normal body temperature and normal blood pressure. Upon physical examination, a grossly distended and diffusely tender abdomen especially in LLQ (Left Lower Quadrant) was revealed. On auscultation abdominal sounds were present. Rectal examination showed an empty rectum. The emergent laboratory tests revealed as follow: WBC $12300 \mathrm{~mm} 3$, hemoglobin at $12.1 \mathrm{~g} / \mathrm{dL}$, creatinine, electrolytes, tumor markers, U/A, and liver function tests were normal and pregnancy test was negative. On vaginal examination uterus was normal and a large mass in left adnex was palpated. Abdominopelvic sonography revealed a mass of $94 * 90 * 123$ $\mathrm{mm}$ and volume of 541cc with regular thick contour located in the left ovary suggestive of huge cystadenoma or carcinoma of the ovary. (Fig. 1). Color doppler ultrasonography revealed absence of blood stream in cyst wall and remained left ovary parenchyma due to torsion of the left adnex or compressive effect of mass on the ovary. Emergent laparotomy was performed for suspected adnexal torsion. Intra operative findings were a large paraovarian mass with thick wall without any torsion .the mass was apart from the ovary but caused compaction of left ovary. Mass was removed completely and sent for frozen section study. Pathological examination showed the diagnosis of cyst hydatid. The cyst lumen contained scolices and hooklet formation. (Pathology figures attached). Postoperative evaluation with thoracic and cranial CT scanning showed no evidence of cyst hydatid. Abdominal CT scan showed three cystic lesions (73, 53 and $47 \mathrm{~mm}$ in diameter) in the liver. Scattered calcified foci were present at these cysts. The postoperative period was uneventful and the patient was discharged 3 days later, with the advice to receive Albendazole. The patient referred to a general surgeon for management of the hepatic cysts.

\section{Discussion:}

Echinococcosis is a parasitic disease that is produced by Echinococcus granulosus and still represents an important medical problem in many countries. Hydatid disease remains a challenging surgical condition worldwide (5). Even though many infections are acquired in childhood, most cases of liver and lung involvement become symptomatic in adult patients because of the slowly growing nature of the cysts. Echinococcosis in extrahepatic sites is usually asymptomatic unless the cyst causes symptoms due to pressure, as in our case, or ruptures to the peritoneal cavity $(6,7)$ Also pelvic hydatid disease can present with vague abdominal pains due to irritation, swelling, menstrual irregularities, infertility and pressure symptoms involving the adjacent organs (vascular structures, ovary, ureters, bladder and rectum) $(3,4,6,7)$. In our case patient the ovary and the paraovarian regions are very 
rare locations for the hydatid cyst disease. The symptoms of hydatid disease are determined by the size, the site, and the condition of the cysts. Echinococcal cysts at unusual locations many times pose diagnostic dilemmas, and the diagnosis sometimes is made intraoperatively as our case (8). We found in literature some case reports presenting unusual location of hydatidosis, but it seems that only few cases of abdominal echinococcosis simulating acute abdomen have been published to date $(9,10)$. It is confirmed that echinococcosis can be found in every organ of the body and that must be considered in the differential diagnosis of acute abdomen, especially in endemic areas. Additional measures, such as the education of farmers and general public, are essential in the control of this zoonotic infection in animals and humans (11). Surgical treatment can be either radical or conservative. Whenever possible, total cystectomy is the gold standard for hydatid cyst treatment. For peritoneal cysts firmly attached to intraperitoneal viscera, uncovering and drainage has been proven to be a safe method. It is important that the abdominal cavity is isolated with gauze soaked in $20 \%$ hypertonic saline solution to avoid secondary hydatosis and allergic reaction (11). The most common complications are generalized toxic reaction due to cyst hydatid rupture and secondary infections. Pre-operative medical treatment reduces the cyst tension, to sterilize the cyst, and to prevent anaphylactic reaction. Postoperative adjuvant Albendazole and Mebendazole medical treatment prevents recurrence. Primary ovarian and paraovarian cyst hydatid disease is a very rare entity. Multiple pelvic masses with abnormal radiologic findings, primary ovarian and pelvic cyst hydatid disease should be considered in the differential diagnosis in endemic geographic regions.

\section{Conflicts of interest:}

Authors declare that there is no any conflict of interest.

\section{Acknowledgments:}

We acknowledge the kind personnel of Women's Reproductive Health Research center.

Figure 1. Abdominopelvic sonography revealed a mass with regular thick contour located in the left ovary.
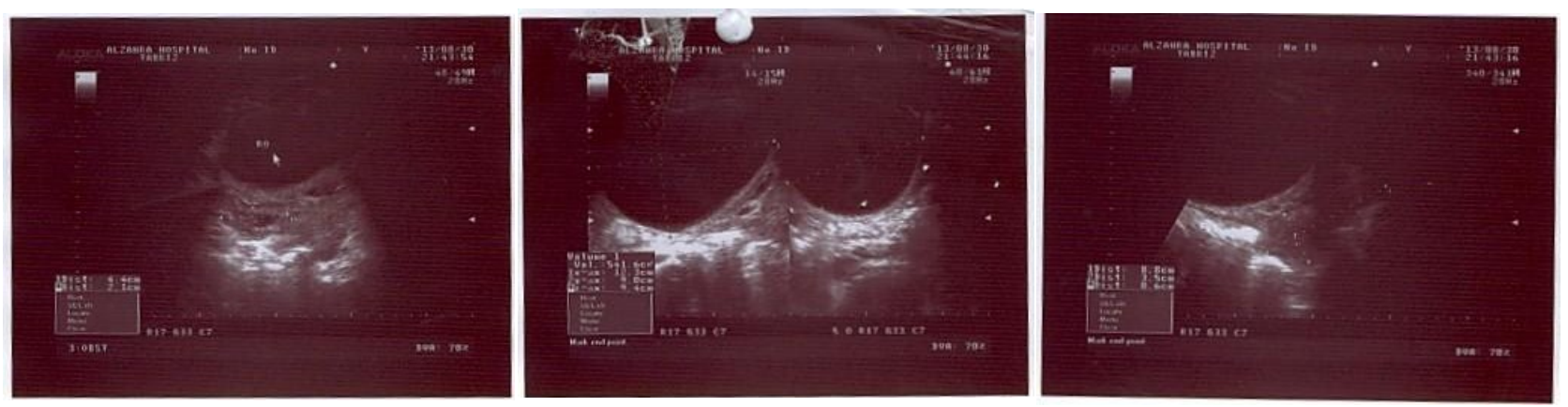

\section{Figure 2.}

A) Low power view of the cyst wall showing laminated appearance (H \& E 100X).

B) Germinative layer of hydatid cyst wall (H \& E 400X).

C) Germinative layer with overlying daughter cysts (H \& E 400X).
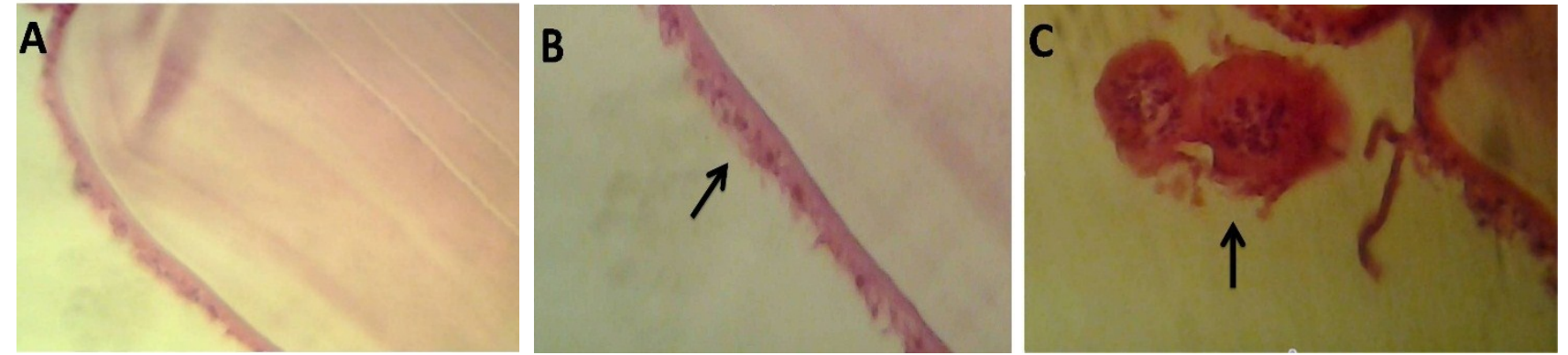


\section{References:}

1. McManus DP, Thompson RC. Molecular epidemiology of cystic echinococcosis. Parasitology. 2003; 127 Suppl:S37-51.

2. Grosso G, Gruttadauria S, Biondi A, Marventano S, Mistretta A. Worldwide epidemiology of liver hydatidosis including the Mediterranean area. World J Gastroenterol. 2012 7; 18(13):1425-37.

3. Moro P, Schantz PM. Echinococcosis: a review. Int J Infect Dis. 2009; 13(2):125-33.

4. Lianos G, Baltogiannis G, Lazaros A, Vlachos K. Hydatid disease simulating acute abdomen: a case report and brief review of the literature. Case Rep Gastrointest Med. 2012; 2012:387102.

5. Tepetes K, Christodoulidis G, Spryridakis M, Hatzitheofilou K. Large solitary retroperitoneal echinococcal cyst: a rare case report. World J Gastroenterol. 2007 7; 13(45):6101-3.

6. Prousalidis J, Tzardinoglou K, Sgouradis L, Katsohis C, Aletras H. Uncommon sites of hydatid disease. World J Surg 1998; 22(1):17-22.

7. Kammerer WS, Schantz PM. Echinococcal disease. Infect Dis Clin North Am. 1993; 7(3):605-18.

8. De U. Primary abdominal hydatid cyst presenting in emergency as appendicular mass: a case report. World J Emerg Surg. 2009 3; 4:13.

9. Ahmad SS, Hassan MJ, Anees A, Rahman K, Zaheer S, Qaseem SM. Hydatid disease of the intestine manifesting as acute abdomen: a case report. Am J Gastroenterol.2010; 105(4):9612 .

10. Sotiraki S, Chaligiannis I. Cystic echinococcosis in Greece. Past and present. Parasite 2010; 17(3):205-10.

11. Ray S, Gangopadhyay M. Hydatid cyst of an ovary: a rare entity. J Turkish-German Gynecol Assoc. 2010; 11: 63-4. 